\title{
openheart Diastolic-systolic velocity ratio to detect coronary stenoses under physiological resting conditions: a mechanistic study
}

\author{
Guus A de Waard, ${ }^{\oplus 1,2}$ Christopher J Broyd, ${ }^{2}$ Christopher M Cook, ${ }^{2}$ \\ Nina W van der Hoeven, ${ }^{1}$ Ricardo Petraco, ${ }^{2}$ Sukhjinder S Nijjer, ${ }^{2}$ \\ Tim P van de Hoef, ${ }^{3}$ Mauro Echavarria-Pinto, ${ }^{4,5}$ Martijn Meuwissen, ${ }^{6}$ Sayan Sen, ${ }^{2}$ \\ Paul Knaapen, ${ }^{1}$ Javier Escaned, ${ }^{4}$ Jan J Piek, ${ }^{3}$ Niels van Royen, ${ }^{1,7}$ Justin E Davies ${ }^{2}$
}

\begin{abstract}
- Additional material is published online only. To view please visit the journal online (http://dx.doi.org/10.1136/ openhrt-2018-000968).
\end{abstract}

To cite: de Waard GA, Broyd CJ, Cook CM, et al. Diastolic-systolic velocity ratio to detect coronary stenoses under physiological resting conditions: a mechanistic study. Open Heart 2019;6:e000968. doi:10.1136/ openhrt-2018-000968

Received 16 November 2018 Revised 27 December 2018 Accepted 20 January 2019

\section{Check for updates}

(c) Author(s) (or their employer(s)) 2019. Re-use permitted under CC BY-NC. No commercial re-use. See rights and permissions. Published by BMJ.

'Department of Cardiology, VU University Medical Center, Amsterdam, The Netherlands ${ }^{2}$ Imperial College London, London, UK

${ }^{3}$ Academic Medical Center, Amsterdam, The Netherlands ${ }^{4}$ Cardiovascular Institute, Hospital Clínico San Carlos, Madrid, Spain

${ }^{5}$ Instituto de Seguridad y Servicios Sociales de IosTrabajadores del Estado, Mexico City, Mexico

${ }^{6}$ Amphia Hospital, Breda, The Netherlands

${ }^{7}$ Radboud University Medical Center, Nijmegen, The Netherlands

Correspondence to Dr Guus A de Waard; $g$. dewaard@vumc.nl

\section{ABSTRACT}

Objective Diastolic-systolic velocity ratio (DSVR) is a resting index to assess stenoses in the left anterior descending artery (LAD). DSVR can be measured by echocardiographic or intracoronary Doppler flow velocity. The objective of this cohort study was to elucidate the fundamental rationale underlying the decreased DSVR in coronary stenoses.

Methods In cohort 1, simultaneous measurements of intracoronary Doppler flow velocity and pressure were acquired in the LAD of 228 stable patients. Phasic stenosis resistance, microvascular resistance and total vascular resistance (defined as stenosis and microvascular resistance combined) were studied during physiological resting conditions. Stenoses were classified according to severity by strata of 0.10 fractional flow reserve (FFR) units.

Results DSVR was decreased in stenoses with lower FFR. Stenosis resistance was equal in systole and diastole for every FFR stratum. Microvascular resistance was consistently higher during systole than diastole. In lower FFR strata, stenosis resistance as a percentage of the total vascular resistance increases both during systole and diastole. The difference between the stenosis resistance as a percentage of total vascular resistance during systole and diastole increases for lower FFR strata, with an accompanying rise in diastolic-systolic resistance ratio. A significant inverse correlation was observed between DSVR and the diastolic-systolic resistance ratio $(r=0.91$, $\mathrm{p}<0.001)$. In cohort $2(\mathrm{n}=23)$, DSVR was measured both invasively and non-invasively by transthoracic echocardiography, yielding a good correlation $(r=0.82$, $\mathrm{p}<0.001$ ).

Conclusions The rationale by which DSVR is decreased distal to coronary stenoses is dependent on a comparatively higher influence of the increased stenosis resistance on total vascular resistance during diastole than systole.

\section{INTRODUCTION}

The physiological process of coronary autoregulation ensures that coronary blood flow is kept constant across a wide range of coronary perfusion pressures by compensatory

\section{Key questions}

What is already known about this subject?

- Diastolic-systolic velocity ratio (DSVR) is an index that possesses the unique ability to detect left anterior descending coronary artery stenoses during resting conditions without the need for pharmacological vasodilators.

- Previous studies have validated the DSVR and demonstrated its feasibility.

What does this study add?

- The fundamental rationale by which DSVR is lower in coronary stenoses despite being measured under resting conditions has not been described.

- In this study, we have demonstrated that the rationale is dependent on a comparatively higher influence of the increased stenosis resistance on total vascular resistance during diastole than systole.

How might this impact on clinical practice?

- The second key result of our study was the close correlation between invasive and non-invasive echocardiographic DSVR.

- Therefore, DSVR determined by echocardiography could potentially facilitate the diagnosis of coronary artery disease in the left anterior descending artery without the use of pharmacological agents.

vasodilation of the coronary microcirculation. ${ }^{2}$ Through coronary autoregulation, the presence of a coronary stenosis does not alter coronary blood flow unless the stenosis totally or subtotally obstructs the coronary lumen. Typically, a pharmacological vasodilatory agent is required to unmask functionally significant coronary artery disease by flow or perfusion-based methods. Despite coronary autoregulation, the ratio between diastolic and systolic coronary flow velocity (DSVR) measured under physiological resting conditions is actually decreased in the presence of obstructive coronary artery disease. ${ }^{3-11}$ DSVR is an index that can either be measured 
invasively with a Doppler flow velocity guidewire or non-invasively in the left anterior descending artery (LAD) with echocardiography. DSVR assessed by echocardiography could potentially represent an inexpensive, quick and broadly available application to detect LAD stenoses without exposing patients to ionic radiation, contrast medium or pharmacological stress agents in specific clinical settings. However, the fundamental rationale to explain the apparent paradox between coronary autoregulation maintaining flow at a stable level and the ratio between diastolic and systolic flow falling distal to a coronary stenosis is presently unknown. Moreover, DSVR has only been investigated in smaller studies, and has not been tested against invasive measurements of functional stenosis severity such as the guideline-recommended fractional flow reserve (FFR) measurement. ${ }^{12}$

In this study, we examined DSVR using the multicentre Iberian-Dutch-English (IDEAL) collaborators registry. ${ }^{1}$ The IDEAL registry comprises a large collection of combined invasively measured coronary pressure and Doppler flow velocity measurements. This registry provides an opportunity to study the physiological mechanism underlying DSVR since coronary resistance measurements were available (cohort 1). Additionally, we report the diagnostic performance of invasive DSVR compared with the FFR. Furthermore, we studied a separate cohort of patients in whom both invasive and non-invasive measurements of DSVR were acquired (cohort 2), in order to explore whether our findings translate to DSVR assessed non-invasively by transthoracic echocardiography.

\section{METHODS}

Cohort 1 used the IDEAL collaborators registry, which involves 301 patients undergoing elective coronary angiography for suspected symptomatic coronary artery disease in four European academic hospitals. ${ }^{1}$ The exclusion criteria for IDEAL were significant valvular disease, previous coronary artery bypass surgery, acute heart failure, acute myocardial infarction within 48 hours of the procedure and prior anterior wall myocardial infarction. Measurements were not acquired in vessels with angiographically identifiable myocardial bridging or collateral arteries. Cohort 2 represents a separate cohort of patients with chest pain and positive functional test scheduled for elective coronary angiography, enrolled in a previous study. ${ }^{13}$ The exclusion criteria for this study were known ischaemic heart disease, valvular pathology, evidence of regional wall motion abnormalities and renal impairment (creatinine $>120 \mu \mathrm{mol} / \mathrm{L}$ ). In cohort 2 , both invasive and non-invasive Doppler measurements of coronary flow velocity were obtained. For both cohorts, only measurements in the $\mathrm{LAD}$ were used since the $\mathrm{LAD}$ is the only coronary artery in which echocardiographic DSVR can be reliably assessed. ${ }^{10}$ In cohort 1,228 patients were included since measurements were taken in the LAD in 228 of 301 patients $(76 \%)$ in the IDEAL registry.

\section{Invasive flow velocity measurements}

Coronary angiography was performed according to standard procedures. After angiography, a 0.014-inch guidewire equipped with both a distal pressure sensor and Doppler crystal (ComboWire XT, Philips Volcano, San Diego, USA) was inserted into the LAD. In the coronary ostium, the pressure sensor was equalised with the pressure of the aortic guiding catheter. Then, the wire was advanced beyond the stenosis, or beyond the proximal segment of the LAD if there was no stenosis. Doppler flow velocity, distal coronary pressure and aortic pressure were measured under true resting conditions. In cohort 1, 200-300 $\mu \mathrm{g}$ of intracoronary nitrates were administered prior to the resting measurements and the measurements were repeated during pharmacological hyperaemia, induced either by intracoronary injection of adenosine $(60-150 \mu \mathrm{g})$ or intravenous adenosine administration $(140 \mu \mathrm{g} / \mathrm{kg} / \mathrm{min})$. In cohort 2 vasodilator drugs were not administered. ${ }^{13}$ Pressure drift was assessed at the end of the procedure, and if pressure drift was identified $(>2 \mathrm{~mm} \mathrm{Hg}$ ) measurements were repeated or corrected for during offline analysis.

\section{Non-invasive echocardiography measurements}

In cohort 2, transthoracic echocardiography was performed as described in an earlier study. ${ }^{13}$ In brief, a Philips ie33 (Amsterdam, The Netherlands) or Esaote MyLab Twice (Genova, Italy) device was used for echocardiography. Starting in the parasternal long axis view, the probe was rotated clockwise and moved laterally across the chest wall until the $\mathrm{LAD}$ was clearly in view with an angulation of $<20^{\circ}$ to the probe. Pulse-wave Doppler was applied with a sampling width of 7.5-10 $\mathrm{mm}$ to record coronary flow velocity signals. Data were exported as high-resolution images and digitalised for data analysis using a MATLAB algorithm (MathWorks, Natick, Massachusetts, USA) with smoothing by a Savitzky-Golay filter.

\section{Data analysis}

For cohort 1, quantitative coronary angiography (using either CAAS II, Pie Medical, Maastricht, The Netherlands; or McKesson, San Francisco, USA) was performed in angiographic stenoses to quantify diameter stenosis percentage, minimal and reference lumen diameter, area stenosis, minimal and reference lumen area, and stenosis length. For both cohorts, data were analysed using an automated MATLAB script (MathWorks) as previously described. ${ }^{14}$ Phasic analysis yielded average values of aortic pressure, distal coronary pressure and average peak Doppler flow velocity for the whole cardiac cycle, systole specifically and mid-to-late diastole specifically. Systole was identified starting at the R peak on the ECG and ending at the dicrotic notch on the aortic pressure trace. Mid-to-late diastole corresponded with the wavefree period, which starts at $25 \%$ of diastole, as marked by the aortic dicrotic notch, and ends 5 ms before systole. ${ }^{145}$ Both invasively and non-invasively measured DSVRs were calculated as the ratio between time-averaged mid-to-late 


\section{A) LAD of a 61 year old female patient with a rest DSVR of 2.4, without a focal stenosis and FFR of 0.99}

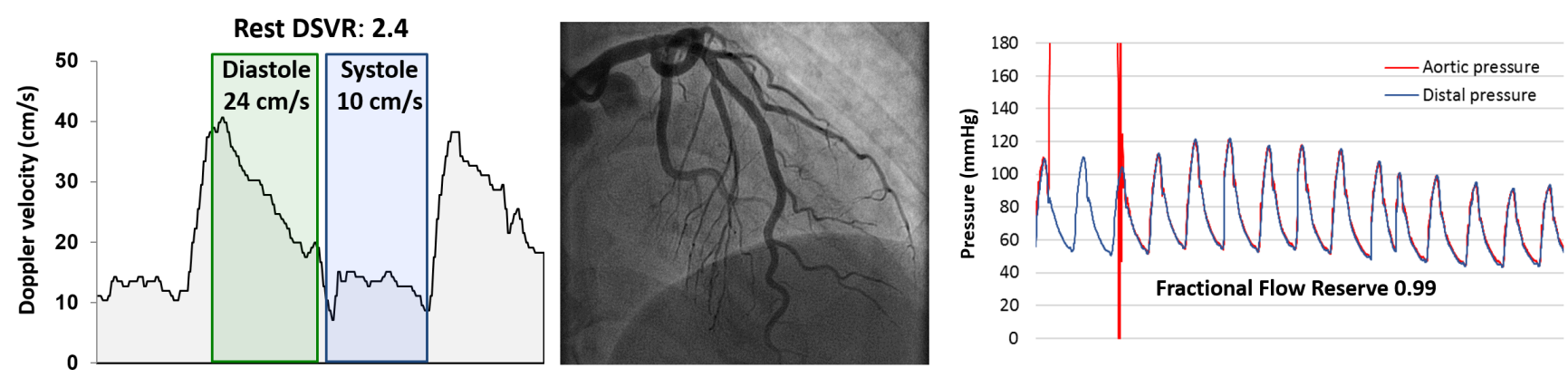

B) LAD of a 58 year old male with a rest DSVR of 1.6, an angiographically significant stenosis and FFR of 0.71
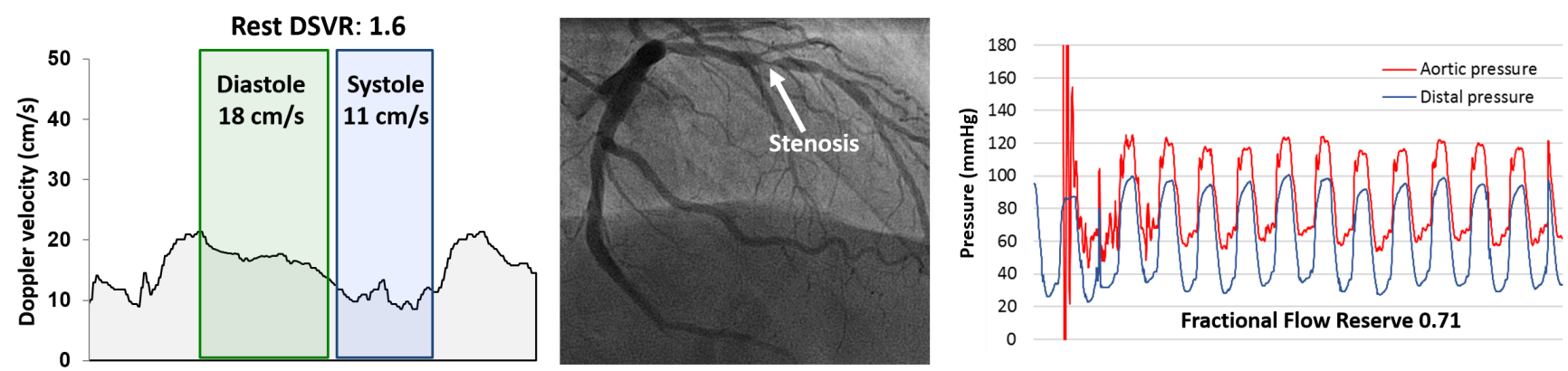

Figure 1 Examples of two patients included in the study. Shown are the Doppler flow velocity measurements during resting conditions (Panel A), the coronary angiogram (Panel B) and the pressure tracing during hyperaemic conditions after injection of $150 \mathrm{\mu g}$ of adenosine (Panel C). DSVR, diastolic-systolic velocity ratio; FFR, fractional flow reserve; LAD, left anterior descending artery.

diastolic and systolic peak Doppler flow velocity. Figure 1 provides two exemplary cases along with the angiogram and hyperaemic pressure tracings. Formulas used for the calculations of all parameters used in this study are shown in box 1. DSVR, stenosis resistance, microvascular resistance, total vascular resistance and diastolic-systolic resistance ratio were calculated during the resting state. FFR $^{16}$ was calculated during the hyperaemic state.

\section{Box 1 Calculations}

$$
\begin{aligned}
& \text { Resting conditions } \\
& D S V R=\frac{\text { Resting diastolic time-averaged Doppler peak velocity }}{\text { Resting systolic time-averaged Doppler peak velocity }} \\
& \text { Resting stenosis resistance }=\frac{\text { Aortic pressure }- \text { distal pressure }}{\text { Average Doppler peak velocity }} \\
& \text { Resting microvascular resistance }=\frac{\text { Distal pressure }}{\text { Average Doppler peak velocity }} \\
& \text { Resting total vascular resistance }=\frac{\text { Aortic pressure }}{\text { Average Doppler peak velocity }} \\
& \text { Resting diastolic }- \text { systolic resistance ratio }=\frac{\text { Diastolic total vascular resistance }}{\text { Systolic total vascular resistance }} \\
& \text { Hyperaemic conditions } \\
& \text { Hyperemic FFR }=\frac{\text { Hyperemic distal pressure }}{\text { Hyperemic aortic pressure }}
\end{aligned}
$$

DSVR, diastolic-systolic velocity ratio; FFR, fractional flow reserve.
Stenosis resistance, microvascular resistance and total vascular resistance were examined during both diastole and systole specifically. FFR was used as reference standards to test the diagnostic accuracy of DSVR, using the FFR threshold of 0.75 , which corresponds best with myocardial ischaemia. ${ }^{16} 17$ For cohort 1 both pressure and Doppler flow velocity data were analysed, thereby also yielding resistance data. For cohort 2, only Doppler flow velocity data were available, precluding calculation of resistance values.

\section{Statistical analysis}

Categorical data are presented as numbers and percentages. Continuous data are presented as mean $\pm \mathrm{SD}$ or median with IQR according to normality of the data. To analyse the diagnostic performance of DSVR, receiver operating characteristic analysis was performed. The optimal DSVR cut-off value was defined as the value with the highest combined specificity and sensitivity. Analysis of variance was used to test for differences across multiple groups. Bonferroni correction was applied for comparison across multiple groups. Linear regression was performed to test the association between two continuous variables. $\log$ transformation was applied to independent and dependent variables in order to achieve linearity of the relationship as required for linear regression analysis. Intraclass correlation coefficients were calculated using a two-way mixed model. A two-sided $p$ value of $<0.05$ was 


\begin{tabular}{llc}
\hline Table 1 & Patient characteristics & \\
Variable & $\begin{array}{l}\text { Cohort 1 } \\
\text { 228 patients }\end{array}$ & $\begin{array}{c}\text { Cohort 2 } \\
\text { 23 patients }\end{array}$ \\
\hline Age (years) & $60.3 \pm 9.7$ & $61.0 \pm 11.0$ \\
Male gender & $163(71)$ & $16(70)$ \\
Female gender & $65(29)$ & $7(30)$ \\
Hypertension & $117(51)$ & $10(43)$ \\
Hypercholesterolaemia & $131(58)$ & $12(52)$ \\
Smoking history & $88(39)$ & $1(4)$ \\
Diabetes mellitus & $49(22)$ & $1(4)$ \\
Previous myocardial infarction & $18(8)$ & $0(0)$ \\
Aspirin & $133(58)$ & $14(61)$ \\
Beta-blocker & $102(45)$ & $3(13)$ \\
Statin & $110(48)$ & $14(61)$ \\
Angiotensin-converting enzyme or & $33(15)$ & $6(26)$ \\
angiotensin-II inhibitor & & $3(13)$ \\
Calcium channel blocker & $38(17)$ & \\
\hline
\end{tabular}

Values are number (percentages) or mean \pm SD.

considered statistically significant. Statistical analyses were performed using SPSS V.22.0.

\section{RESULTS}

\section{Study population}

In this study, two cohorts were used, and for both cohorts patient characteristics are shown in table 1 . Cohort 1 consisted of 228 patients with a mean age of $60.3 \pm 9.7$ years and of whom 163 were male $(71 \%)$ and 65 were female $(29 \%)$. For the invasive cohort, vessel and stenosis characteristics are shown in table 2 . One hundred and fiftythree $(67 \%)$ measurements were taken in a vessel with an angiographic stenosis and $75(33 \%)$ in unobstructed reference vessels. In cohort 2 , consisting of patients in whom both invasive and non-invasive measurements of DSVR were obtained, 24 subjects were included. Of these 24 subjects, 1 had to be excluded because of poor echocardiographic Doppler flow envelopes precluding DSVR calculation, leaving 23 for final analysis. The mean age of these 23 patients was $61.0 \pm 11.0$ years, of whom 16 were male $(70 \%)$ and 7 were female $(30 \%)$.

\section{Cohort 1: invasive DSVR according to stenosis severity}

Figure 2 shows invasive DSVR classified according to FFR values as 0.10 unit groups. In LAD branches with FFR $>0.95$, a DSVR of $2.10 \pm 0.42$ is observed that gradually decreases to $1.55 \pm 0.25$ with FFR $<0.65$ ( $\mathrm{p}_{\text {trend }}<0.001$ ). Online supplementary figure 1 shows the correlation between FFR and DSVR. Similar observations are made when DSVR is classified according to the instantaneous wave-free ratio and to the hyperaemic stenosis resistance index instead of FFR (online supplementary figures 2 and 3 , respectively). Figure 3 shows the receiver operating characteristic curve for invasive DSVR to predict FFR at

\begin{tabular}{|c|c|}
\hline Variable & \\
\hline Intracoronary adenosine & $121(53 \%)$ \\
\hline Intravenous adenosine & $107(47 \%)$ \\
\hline Unobstructed vessels & 75 (33\%) \\
\hline FFR & 0.94 (IQR 0.90-0.98) \\
\hline DSVR & $2.08 \pm 0.47$ \\
\hline Resting APV (cm/s) & 16.1 (IQR 12.3-22.1) \\
\hline Diastolic APV (cm/s) & 21.1 (IQR 16.3-29.5) \\
\hline Systolic APV (cm/s) & 10.7 (IQR 7.8-15.6) \\
\hline Coronary flow reserve & $2.80 \pm 0.86$ \\
\hline Obstructed vessels & $153(67 \%)$ \\
\hline Minimal lumen diameter (mm) & $1.18 \pm 0.51$ \\
\hline Reference diameter (mm) & $2.73 \pm 0.75$ \\
\hline Diameter stenosis \% & $55 \pm 15$ \\
\hline Minimal area stenosis $\left(\mathrm{mm}^{2}\right)$ & 0.98 (IQR 0.57-1.63) \\
\hline Reference area $\left(\mathrm{mm}^{2}\right)$ & 5.5 (IQR 3.7-7.9) \\
\hline Area stenosis \% & 79 (IQR 70-88) \\
\hline Stenosis length (mm) & 16 (IQR 8.0-24) \\
\hline FFR & 0.81 (IQR 0.65-0.88) \\
\hline DSVR & $1.72 \pm 0.39$ \\
\hline Resting APV (cm/s) & 15.9 (IQR 11.4-21.4) \\
\hline Diastolic APV (cm/s) & $19.7(14.6-28.4)$ \\
\hline Systolic APV (cm/s) & $12.0(8.8-16.8)$ \\
\hline Coronary flow reserve & $2.03 \pm 0.83$ \\
\hline
\end{tabular}

Values are number (percentages), median (IQR) or mean $\pm S D$. APV, average peak velocity; DSVR, diastolic-systolic velocity ratio; FFR, fractional flow reserve.

its ischaemic threshold with a C-statistic of $0.76(95 \%$ CI 0.69 to 0.82 ). The optimal cut-off value for DSVR was 1.74 , yielding a sensitivity of $65 \%$ and specificity of $80 \%$.

\section{Cohort 1: phasic coronary resistance}

Figure 4 shows the relationship between resting flow velocity and total vascular resistance for diastole (figure 4A) and systole (figure 4B). Strong inverse relationships between flow velocity and vascular resistance are observed for both phases $(\mathrm{r}=0.95, \mathrm{p}<0.001$ for diastole, and $r=0.93, p<0.001$ for systole). Figure $4 \mathrm{C}$ shows the relationship between DSVR and the diastolic-systolic resistance ratio. In line with the inverse relationships between the diastolic and systolic components of these ratios, a strong inverse relationship between DSVR and the diastolic-systolic resistance ratio is also observed: $r=0.91, \mathrm{p}<0.001$. Because of the strong correlation between DSVR and the diastolic-systolic resistance ratio, we used resistance data to further explore the potential mechanism driving the lower DSVR in functionally significant stenoses. Figure 5 shows the resting vascular resistance for both the microvascular and stenosis components stratified according to FFR values. Figure $5 \mathrm{~A}$ shows that microvascular resistance 


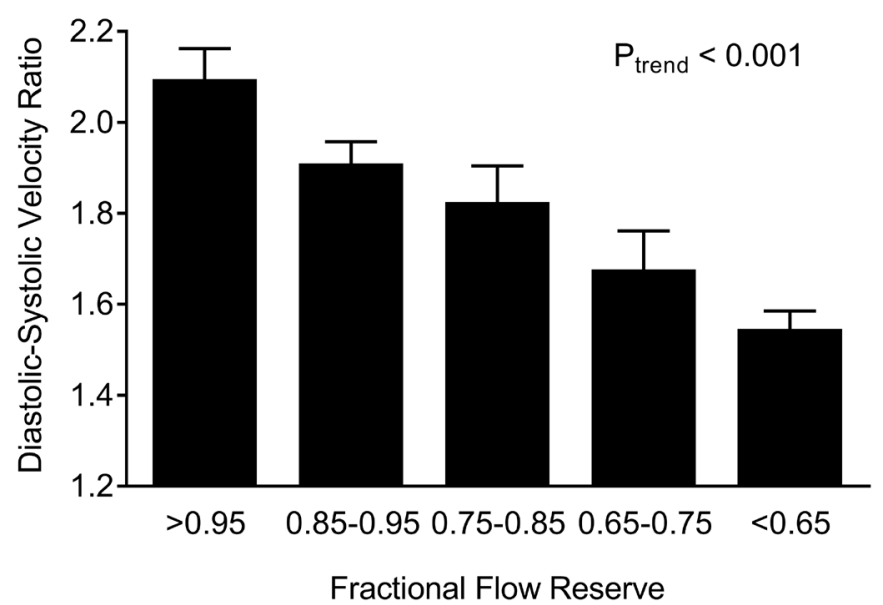

Figure 2 Invasive diastolic-systolic velocity ratio according to fractional flow reserve classification in cohort 1. Bars represent mean and error bars represent SEM.

is consistently significantly lower during diastole than systole for each FFR group $(\mathrm{p}<0.001$ for all). In contrast, Figure $5 \mathrm{~B}$ shows that stenosis resistance is generally not significantly different between diastole and systole ( $p>0.05$ for all, apart from the FFR $0.85-0.95$ group with $\mathrm{p}<0.05)$. Figure $5 \mathrm{C}$ integrates the previous panels to show the contribution of microvascular and stenosis resistance to overall vascular resistance for diastole and systole in each FFR group. Figure 5D depicts the stenosis resistance as a percentage of total resistance classified according to FFR value for both diastole and systole. It can be observed that stenosis resistance represents a higher percentage of total vascular resistance during diastole than during

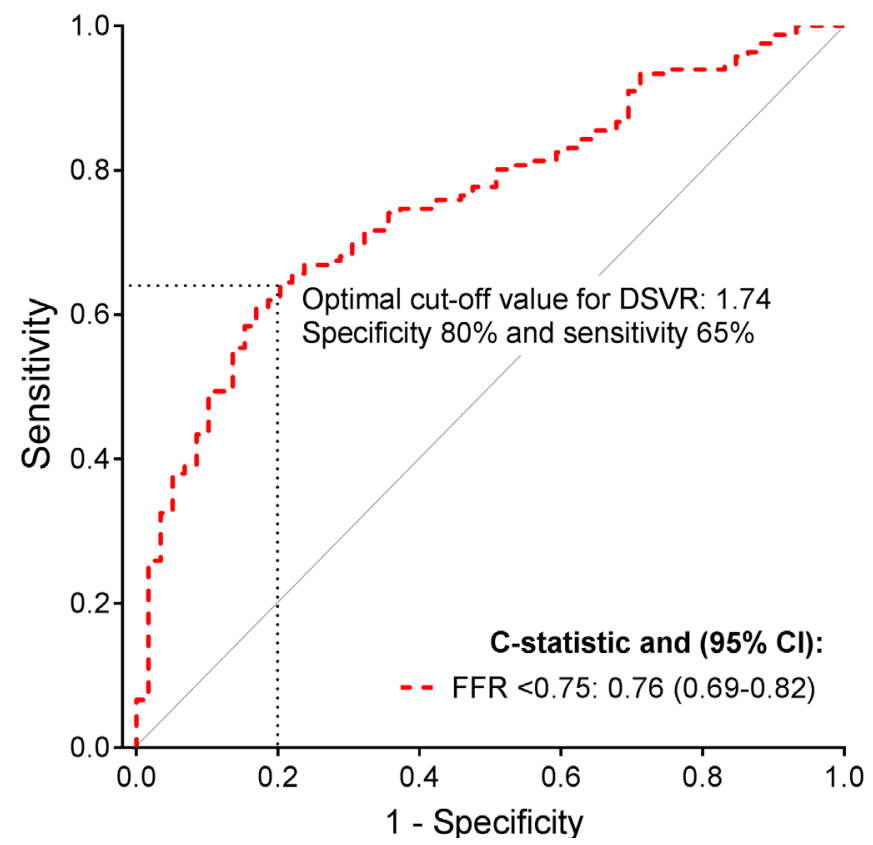

Figure 3 Diagnostic performance of invasive DSVR in cohort 1. Receiver operating characteristic curves for DSVR in the left anterior descending artery branches to predict FFR at its ischaemic threshold of 0.75. DSVR, diastolic-systolic velocity ratio; FFR, fractional flow reserve. systole for all FFR groups $(\mathrm{p}<0.001$ for all apart from the FFR $>0.95$ group). Figure $5 \mathrm{E}$ shows the difference between diastolic and systolic stenosis resistance as a percentage of total vascular resistance according to FFR. A gradual increase in the difference between diastolic and systolic stenosis resistance as a percentage of total vascular resistance is observed as FFR worsens $\left(\mathrm{p}_{\text {trend }}<0.001\right)$. Finally, Figure $5 \mathrm{~F}$ shows that a gradual increase of the diastolic-systolic resistance ratio occurs with worsening of FFR group $\left(\mathrm{p}_{\text {trend }}<0.001\right)$. Together, Figure 5 shows that with increasing functional stenosis severity, the stenosis resistance has greater impact on total vascular resistance during diastole than systole. Because DSVR is inversely related to the diastolic-systolic vascular resistance ratio, this observation likely extends to DSVR and explains why DSVR is decreased in more severe stenoses (figure 6).

Cohort 2: correlation between invasive and non-invasive DSVR In this paragraph, the results are described for the patients in cohort 2 with paired invasive and non-invasive echocardiographic measurements. Table 3 describes haemodynamic data for both the invasive and non-invasive echocardiographic measurements. A reasonable correlation was observed between invasive and non-invasive DSVR: $r=0.82, p<0.001$, with a good intraclass correlation coefficient of 0.80 (95\% CI 0.59 to 0.91$)$, and a small mean difference of $0.11 \pm 0.24$ DSVR units (figure 7).

\section{DISCUSSION}

In this study, we investigated the theoretical rationale of DSVR and sought to determine the concordance between invasively and non-invasively measured DSVR. The following are our findings: (1) Invasive DSVR has reasonable diagnostic properties to detect functionally significant stenoses in the LAD compared with FFR. (2) The rationale to explain why DSVR in the $\mathrm{LAD}$ corresponds to functional stenosis severity is related to a progressively higher impact of stenosis resistance on diastole than on systole with worsening of functional stenosis severity. (3) Invasively and non-invasively measured DSVRs correlate well with one another. The interpretation, potential clinical implications and limitations of our work are discussed below.

\section{Diagnostic accuracy of DSVR}

Several smaller previous studies investigating DSVR, measured invasively or by echocardiography, documented acceptable diagnostic accuracy when compared with thallium scintigraphy, coronary angiography and echocardiographic coronary flow velocity reserve. 57910 Our study adds to the existing body of evidence that invasively measured DSVR in the LAD provides reasonable diagnostic accuracy when compared with FFR at its ischaemic threshold.

\section{Rationale underlying DSVR}

Although the diagnostic performance of DSVR was already explored in previous studies, the rationale behind 
A

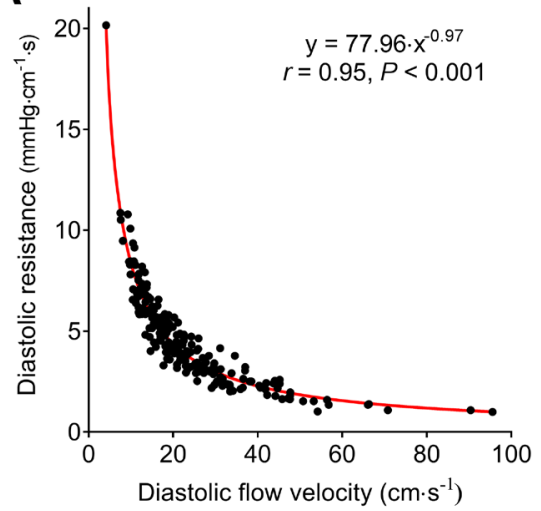

B

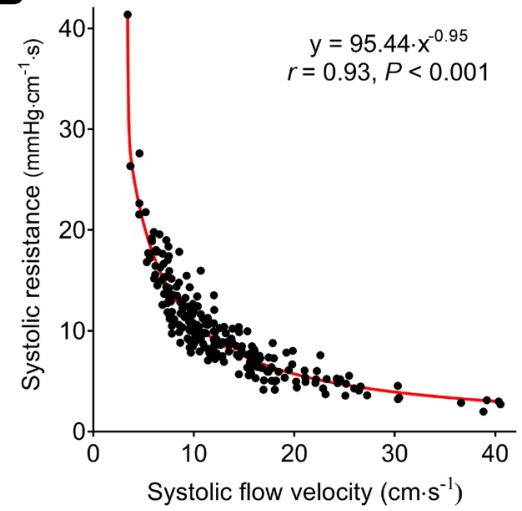

C

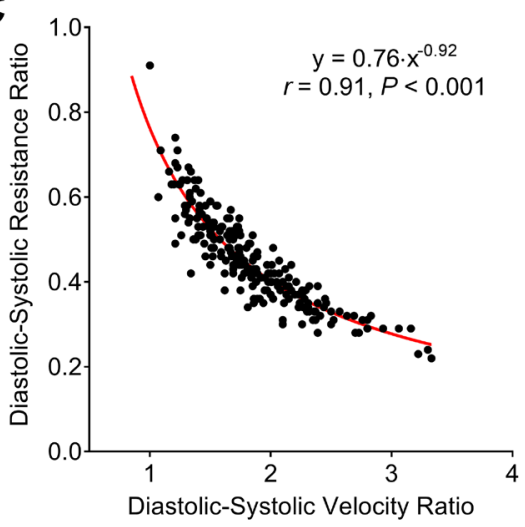

Figure 4 Relationship between invasive phasic flow and resistance in cohort 1. Panel A shows a strong inverse relationship between diastolic flow velocity and diastolic vascular resistance. Panel B shows similar results for systole. Panel C demonstrates a strong inverse relationship between DSVR and the ratio between diastolic and systolic vascular resistance. DSVR, diastolic-systolic velocity ratio.

DSVR remained hypothetical as tangible evidence was lacking. ${ }^{4}$ It may seem counterintuitive that the functional significance of a stenosis can be assessed from resting coronary blood flow, since coronary autoregulation keeps resting mean flow at a stable plateau despite pressure losses incurred by the stenosis. ${ }^{12}$ Nevertheless, our results confirm that invasive DSVR decreases gradually alongside worsening of functional stenosis significance. Because phasic distal coronary flow velocity data as well as proximal and distal coronary pressure data are available in the IDEAL registry, stenosis resistance and microvascular resistance could be distinguished for both diastole and systole. Therefore, we were able to establish the theoretical rationale by which DSVR informs on functional stenosis significance in the LAD. The finding that DSVR is inversely related to the diastolic-systolic vascular
A

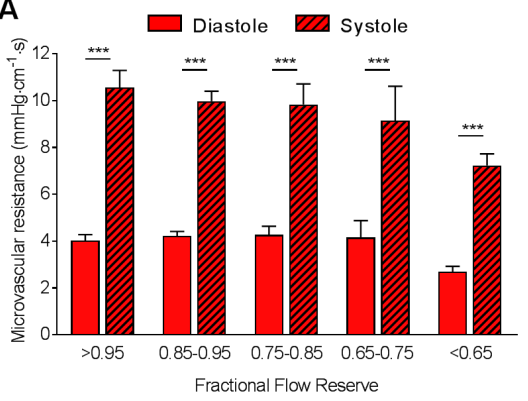

D

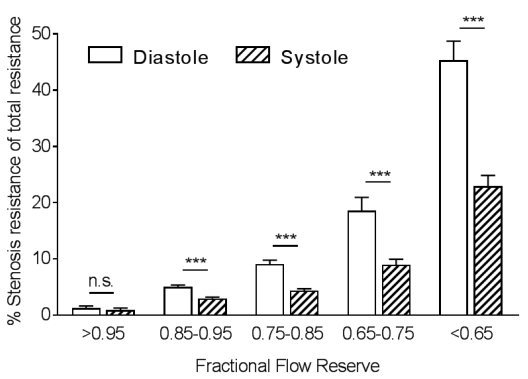

B

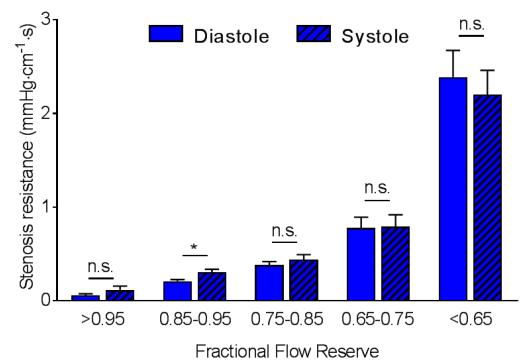

E

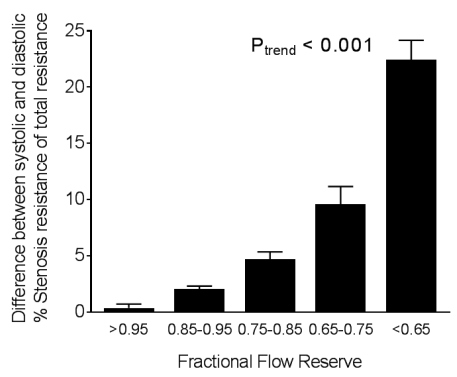

C

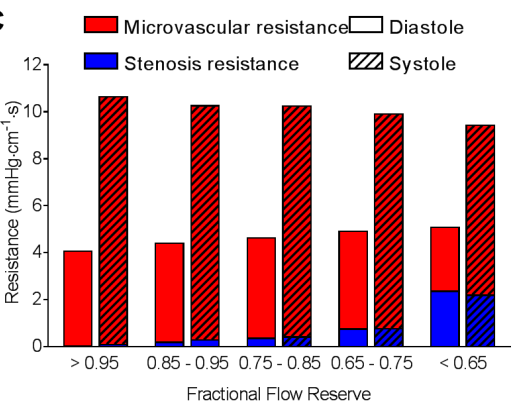

$\mathbf{F}$

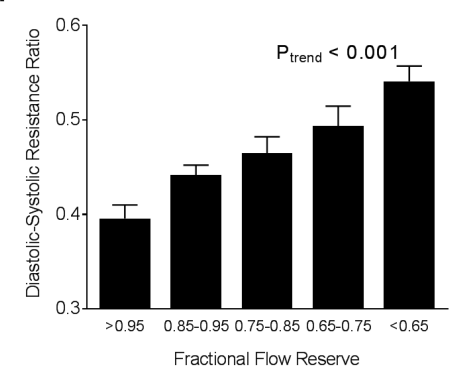

Figure 5 Invasive resistance distribution according to functional stenosis severity in cohort 1. Panel A shows the distribution of microvascular resistance stratified according to diastole (open bars) and systole (marked bars) classified by FFR. Panel B shows the distribution of stenosis resistance stratified according to diastole (open bars) and systole (marked bars) classified by FFR. Panel $\mathrm{C}$ shows the total resistance per FFR group stratified according to systole and diastole by integrating microvascular (red bars) and stenosis resistance (blue bars). Panel D shows the stenosis resistance as a percentage of the total vascular resistance composed of both stenosis and microvascular resistance for both diastole (open bars) and systole (marked bars). Panel E shows the difference between the diastolic and systolic percentage stenosis resistance of total resistance. As FFR group worsens, the stenosis resistance constitutes a comparatively greater portion of total resistance for diastole than for systole. Finally, panel F shows that the ratio between diastolic and systolic vascular resistance increases as FFR worsens. Bars represent mean and error bars represent SEM. *Indicates $p$ is significant after Bonferroni correction, ${ }^{* *}$ indicates $p<0.001$ and NS indicates $\mathrm{p}$ is non-significant after Bonferroni correction. FFR, fractional flow reserve. 
Diastole

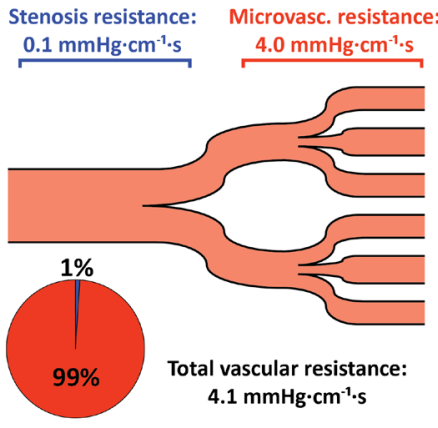

\section{Systole}

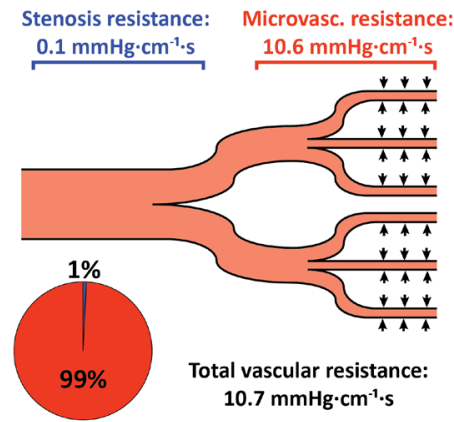

Diastolic-systolic resistance ratio: $\mathbf{0 . 3 9}$

B

\section{Severe LAD stenosis: FFR $<0.65$ and DSVR 1.5 Diastole \\ Systole}

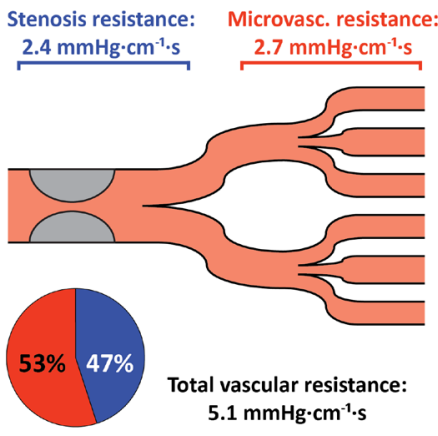

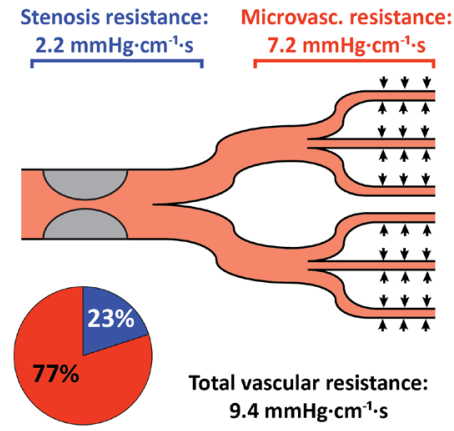

Diastolic-systolic resistance ratio: $\mathbf{0 . 5 4}$

Figure 6 Summary of the DSVR rationale. This figure illustrates the shift in resting resistance distributions during diastole and systole averaged for the group of unobstructed LAD branches with FFR $>0.95$ and DSVR of 2.1 (A) and averaged for the group with obstructed LAD branches with FFR $<0.65$ and DSVR of 1.5 (B). Because stenosis resistance (blue area in the pie charts) is negligible in unobstructed LAD branches, its contribution to total vascular resistance is low during both diastole and systole (both 1\%). In LAD branches with stenosis, the stenosis resistance represents $47 \%$ of total vascular resistance during diastole when the microvasculature is open and microvascular resistance is low (red area in the pie charts). During systole, however, the microvasculature is compressed by the contracting myocardium resulting in high microvascular resistance. Because stenosis resistance does not appreciably differ during diastole and systole, the stenosis resistance has a much smaller impact during systole and only represents $23 \%$ of total vascular resistance. As such, in stenosis the diastolic-systolic resistance ratio is increased compared with unobstructed vessels. DSVR, diastolic-systolic velocity ratio; FFR, fractional flow reserve; LAD, left anterior descending artery.

Table 3 Haemodynamic characteristics of cohort 2

\begin{tabular}{|lll|}
\hline Variable & $\begin{array}{l}\text { Invasive } \\
\text { measurements }\end{array}$ & $\begin{array}{l}\text { Non-invasive } \\
\text { echocardiographic } \\
\text { measurements }\end{array}$ \\
\hline DSVR & $1.96(1.77-2.40)$ & $2.02(1.70-2.18)$ \\
\hline $\begin{array}{l}\text { Resting APV } \\
\text { (cm/s) }\end{array}$ & $24.4(15.6-29.7)$ & $21.5(17.0-27.6)$ \\
\hline $\begin{array}{l}\text { Diastolic APV } \\
\text { (cm/s) }\end{array}$ & $32.0(22.9-42.2)$ & $27.6(22.8-37.5)$ \\
\hline $\begin{array}{l}\text { Systolic APV } \\
\text { (cm/s) }\end{array}$ & $14.7(10.8-20.7)$ & $14.9(11.3-19.2)$ \\
\hline
\end{tabular}

Values are described as median with IQR.

APV, average peak velocity; DSVR, diastolic-systolic velocity ratio. resistance ratio implies that phasic changes of coronary resistances across the stenosis and microvascular compartments underlie the reduced DSVR in obstructive stenoses. Inspection of the resistance distribution presented in figure 5 reveals that microvascular resistance is consistently lower during diastole than systole, but stenosis resistance is generally similar during diastole and systole. Therefore, the stenosis resistance constitutes a higher proportion of total resistance during diastole than during systole. As such, in functionally severe stenoses, the high stenosis resistance has greater impact on total diastolic resistance than on systolic resistance. Consequently, the diastolic-systolic resistance ratio increases as stenosis severity progresses. Finally, because of the inverse relationship between DSVR and the diastolic-systolic 
A

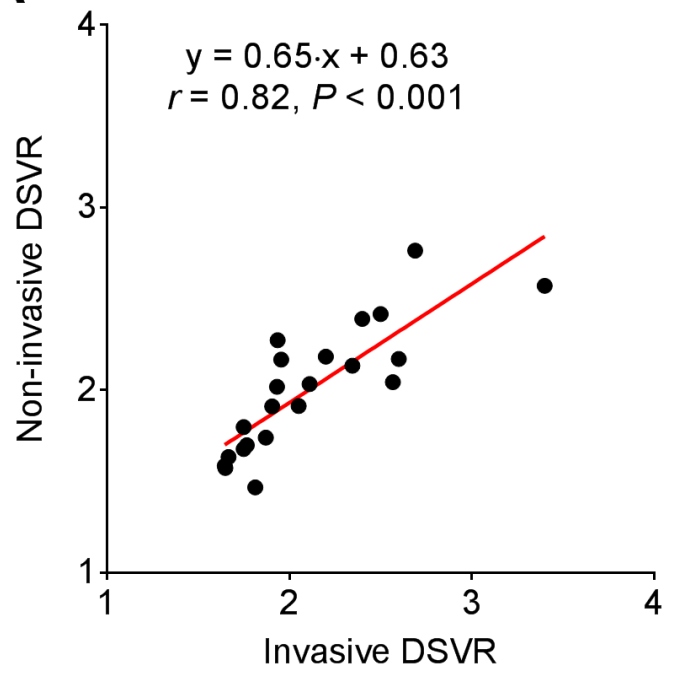

B

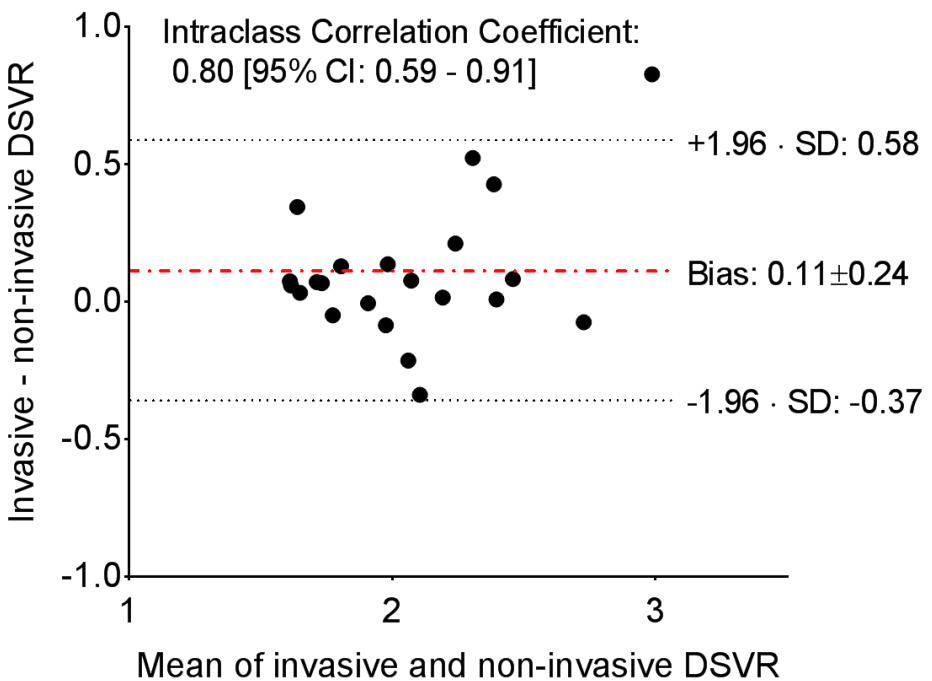

Figure 7 Correlation between invasive and non-invasively measured DSVR in cohort 2. This figure shows DSVR measured both invasively and non-invasively by transthoracic echocardiography within the same patient from the separate cohort. DSVR, diastolic-systolic velocity ratio.

resistance ratio, DSVR decreases with worsening of FFR group (figure 6).

\section{Clinical implications}

In line with earlier studies, ${ }^{5} 7910$ we report that invasive DSVR can be used to identify LAD stenoses responsible for inducible myocardial ischaemia with an optimal cut-off value of 1.74 . These observations could warrant consideration for clinical use of DSVR, specifically for non-invasively measured DSVR. Non-invasively measured DSVR could be useful in certain clinical scenarios to assess the presence of a functionally significant LAD stenosis. For instance, DSVR could be an easily accessible information during routine echocardiography. A second potential application is to use DSVR for patients with previous LAD revascularisation if instent restenosis is suspected. Third, non-invasive measurement of DSVR is also possible in left internal mammary artery bypass grafts. In this context, DSVR could inform on whether or not competitive flow from the LAD occurs. ${ }^{18}$

\section{Limitations}

The present study has a number of limitations. First, despite invasive assessment of coronary Doppler flow velocity, signal quality was not always optimal and $13 \%$ of patients had to be excluded from the original IDEAL registry for this reason. ${ }^{1}$ Although similar concerns exist for echocardiography-derived DSVR, earlier studies have reported success rates of echocardiography-derived DSVR of around $85 \%$ in the LAD. ${ }^{6} 10$ The most frequent reason for failure of DSVR measurement in these studies was total or subtotal obstruction of the LAD, meaning that the immeasurable DSVR in fact correctly identified the absence of coronary flow patterns. Second, we calculated DSVR using time-averaged peak values of Doppler flow velocity, whereas other studies have used single peak values. ${ }^{6910}$ Although using single peak values could be more convenient for clinical practice, it could also be more susceptible to measurement artefacts such as spikes. This hypothesis was confirmed by Daimon et $a l^{7}$, who showed that echocardiographic DSVR calculated using mean values compared with peak values had superior test characteristics to detect thallium scintigraphy-defined myocardial ischaemia. Third, altered myocardial contractility may influence DSVR, but we could not study this issue since pressure and flow assessments were not made in patients with anterior or septal regional wall motion abnormalities. Fourth, although invasively and non-invasively measured DSVR correlated reasonably well, the agreement was not perfect. Possibly, haemodynamic fluctuations in heart rate, blood pressure and myocardial contractibility precluded better agreement. Also, small artefacts in the Doppler flow velocity tracings for both methods of flow velocity assessment could have contributed to the imperfect agreement. Fifth, our study did not address the correlation between DSVR measured by echocardiography and the FFR. Finally, coronary venous pressure, wedge pressure and left ventricular end-diastolic pressure were not available for resistance calculations. However, comorbidities with expected alterations of these pressures such as acute heart failure, angiographically visible collaterals and severe aortic valvular disease were exclusion criteria. Therefore, we assumed that the effects of venous, wedge and left ventricular end-diastolic pressure on coronary pressure and resistance were negligible.

\section{CONCLUSION}

The findings of this study show that the fundamental rationale by which DSVR is able to detect the presence 
of LAD stenoses is because the stenosis resistance has a comparatively greater influence on total vascular resistance during diastole, when microvascular resistance is low, than during systole, when microvascular resistance is high. Because invasively measured DSVR correlates reasonably well with non-invasive DSVR, our findings using invasively measured coronary haemodynamics should hypothetically also apply to non-invasive DSVR.

Contributors GdW, CB, RP, SSN, TPvdH, ME-P, SS, MM, PK, JE, JJP, NvR and JD were involved in data collection. GdW designed the study, performed data and statistical analysis, and drafted the manuscript. NvR and JD supervised the project. All authors made critical revisions to key intellectual content of the manuscript.

Funding A fellowship grant awarded to GdW by the ICIN Netherlands Heart Institute helped fund the present study.

Competing interests JD is codeveloper of intellectual property, which is licensed to Philips Volcano by Imperial College London. He receives consultancy and research funding from Philips Volcano. CC, RP, SSN, JJP, TPvdH, NVR and JE report receiving fees for speaking at educational events organised by Philips Volcano. The other authors report no conflict of interest.

Patient consent for publication Obtained.

Ethics approval The different study protocols were approved by the respective institutional review committees and complied with the principles of the Declaration of Helsinki.

Provenance and peer review Not commissioned; externally peer reviewed.

Data sharing statement № additional data are available.

Open access This is an open access article distributed in accordance with the Creative Commons Attribution Non Commercial (CC BY-NC 4.0) license, which permits others to distribute, remix, adapt, build upon this work non-commercially, and license their derivative works on different terms, provided the original work is properly cited, appropriate credit is given, any changes made indicated, and the use is non-commercial. See: http://creativecommons.org/licenses/by-nc/4.0/.

\section{REFERENCES}

1. Nijer SS, de Waard GA, Sen S, et al. Coronary pressure and flow relationships in humans: phasic analysis of normal and pathological vessels and the implications for stenosis assessment: a report from the Iberian-Dutch-English (ideal) collaborators. Eur Heart $J$ 2016;37:2069-80.

2. de Waard GA, Cook CM, van Royen N, et al. Coronary autoregulation and assessment of stenosis severity without pharmacological vasodilation. Eur Heart J 2018;39:4062-71.

3. Segal J, Kern MJ, Scott NA, et al. Alterations of phasic coronary artery flow velocity in humans during percutaneous coronary angioplasty. J Am Coll Cardiol 1992;20:276-86.

4. Ofili EO, Kern MJ, Labovitz AJ, et al. Analysis of coronary blood flow velocity dynamics in angiographically normal and stenosed arteries before and after endolumen enlargement by angioplasty. J Am Coll Cardiol 1993;21:308-16.
5. Deychak YA, Segal J, Reiner JS, et al. Doppler quide wire flow-velocity indexes measured distal to coronary stenoses associated with reversible thallium perfusion defects. Am Heart $J$ 1995;129:219-27.

6. Crowley JJ, Shapiro LM. Analysis of phasic flow velocity dynamics in the left anterior descending coronary artery before and after angioplasty using transthoracic echocardiography in patients with stable angina pectoris. Am J Cardiol 1997;80:614-7.

7. Daimon $\mathrm{M}$, Watanabe $\mathrm{H}$, Yamagishi $\mathrm{H}$, et al. Physiologic assessment of coronary artery stenosis without stress tests: noninvasive analysis of phasic flow characteristics by transthoracic Doppler echocardiography. J Am Soc Echocardiogr 2005;18:949-55

8. Okura $\mathrm{H}$, Fuyuki $\mathrm{H}$, Kubo $\mathrm{T}$, et al. Noninvasive diagnosis of ischemic and nonischemic cardiomyopathy using coronary flow velocity measurements of the left anterior descending coronary artery by transthoracic Doppler echocardiography. J Am Soc Echocardiogr 2006;19:552-8

9. Tani T, Tanabe K, Kitai T, et al. Detection of severe stenosis and total occlusion in the left anterior descending coronary artery with transthoracic Doppler echocardiography in the emergency room. Echocardiography 2009;26:15-20.

10. Holte E, Vegsundvåg J, Hegbom K, et al. Transthoracic Doppler echocardiography for detection of stenoses in the left coronary artery by use of poststenotic coronary flow profiles: a comparison with quantitative coronary angiography and coronary flow reserve. $J$ Am Soc Echocardiogr 2013;26:77-85.

11. Piek JJ, Boersma E, di Mario C, et al. Angiographical and Doppler flow-derived parameters for assessment of coronary lesion severity and its relation to the result of exercise electrocardiography. debate Study Group. Doppler endpoints balloon angioplasty trial Europe. Eur Heart J 2000;21:466-74.

12. Montalescot G, Sechtem U, Achenbach S, et al. 2013 ESC guidelines on the management of stable coronary artery disease: the task Force on the management of stable coronary artery disease of the European Society of cardiology. Eur Heart J 2013;34:2949-3003.

13. Broyd CJ, Nijjer S, Sen S, et al. Estimation of coronary wave intensity analysis using noninvasive techniques and its application to exercise physiology. Am J Physiol Heart Circ Physiol 2016;310:H619-H627.

14. Sen S, Escaned J, Malik IS, et al. Development and validation of a new adenosine-independent index of stenosis severity from coronary wave-intensity analysis: results of the advise (adenosine vasodilator independent stenosis evaluation) study. J Am Coll Cardiol 2012;59:1392-402.

15. de Waard GA, Di Mario C, Lerman A, et al. Instantaneous wavefree ratio to guide coronary revascularisation: physiological framework, validation and differences from fractional flow reserve. Eurolntervention 2017;13:450-8.

16. Pijls $\mathrm{NH}$, van Son JA, Kirkeeide RL, et al. Experimental basis of determining maximum coronary, myocardial, and collateral blood flow by pressure measurements for assessing functional stenosis severity before and after percutaneous transluminal coronary angioplasty. Circulation 1993;87:1354-67.

17. Pijls NH, De Bruyne B, Peels K, et al. Measurement of fractional flow reserve to assess the functional severity of coronary-artery stenoses. N Engl J Med 1996;334:1703-8.

18. Pizzuto F, Voci P, Mariano E, et al. Evaluation of flow in the left anterior descending coronary artery but not in the left internal mammary artery graft predicts significant stenosis of the arterial conduit. J Am Coll Cardiol 2005;45:424-32. 